Department of Psychiatry (Chairman: Albert J. Silverman, M.D.), University of Michigan, Ann Arbor, Michigan, U.S.A.

\title{
Premenstrual tension syndrome: The development of research diagnostic criteria and new rating scales
}

\author{
M. Steiner, R. F. Haskett and B. J. Carroll
}

\begin{abstract}
Research diagnostic criteria for premenstrual tension syndrome (PMTS) are developed using data collected from a study of 42 women who were suffering from severe PMTS but were well at other times. Two specific scales are also devised for rating the severity of PMTS, a 36-item self-report questionnaire and a 10 item scale for use by therapist/researcher. It is proposed that after further evaluation and validation, these instruments may permit more useful comparisons of data on the etiology and treatment of this disorder.
\end{abstract}

Key words: Premenstrual tension syndrome (PMTS) - research diagnostic criteria (RDC).

In reviewing the extensive literature on premenstrual tension syndrome (PMTS) it is astonishing to find how many pitfalls this "minor", so very common, transient phenomenon presents to the researcher. It afflicts, in a cyclic pattern, many women during their fertile years and is often considered as a normal, natural, or somewhat inconvenient aspect of being female. Yet in some women PMTS is an incapacitating ailment, serious enough to disrupt their lives and warrant treatment. It seems to affect both body and mind and its description encompasses a large array of emotional and physical symptoms (Frank (1931), Altman et al. (1941), Rees (1953), Fortin et al. (1958), Dalton (1964)).

These women are treated by gynecologists and family practitioners, psychiatrists and psychologists. As a result, it is variously categorized as a psychophysiological state, a somato-psychic or a psychosomatic disease, but none of these formulations seem to provide any operational advantage. PMTS has been linked to various hormonal changes or electrolyte imbalances (Rees (1953), Green \& Dalton (1953)) but the precise etiology of these syndromes remains unclear. A long list of treatments and therapies have been suggested but most fade slowly after the initial enthusiasm (Smith (1975), Steiner \& Carroll (1977)). To date, none of the treatments have survived double-blind placebo controlled trials and there is some evidence that PMTS shows a marked placebo response with any therapy (Smith et al. (1975)). The conflicting reports of the relationship between PMTS and other psychiatric disorders only add to the general confusion (Rees (1953), Coppen \& Kessel (1963), Diamond et al. (1976)).

It is readily apparent that there is little agreement on the precise nature, severity and duration of this syndrome. Despite the use of similar descriptive 
titles, it is possible that comparisons are being made between populations with their own peculiar characteristics apart from PMTS, and suffering from quite different disorders during their menstrual cycle. The new Research Diagnostic Criteria (RDC) (Spitzer et al. (1978)), which have been published for many psychiatric syndromes, are proposed as a means of dealing with nosological inconsistency and the research difficulties which follow. They contribute greatly to the recent draft of the DSM-III (American Psychiatric Association (1978)) but in neither system is PMTS recognized as a distinct entity. The Hospital International Classification of Diseases Adaptation (HICDA) (Commission on Professional and Hospital Activities (1973)) mentions PMTS only in the section of gynecologic diseases, under Disorders of Menstrual Cycle (Code No. 626.8). Currently there are no operationally defined criteria for the precise time course and phenomenology of this syndrome. Also, no validated rating scale exists for the specific measurement of severe premenstrual symptomatology. Commonly used scales are the Menstrual Distress Questionnaire (MDQ) (Moos (1969)) and the Multiple Affect Adjective Check List (MAACL) (Zuckerman \& Lubin (1965)). These, however, cover a broader range of phenomena and are less helpful in measuring the particular changes which occur in PMTS. We felt that before any further attempts were made to link the physiology of menstruation to PMTS or to study the etiology, pathophysiology and treatment of PMTS, there was an urgent need for valid criteria to diagnose this syndrome and for instruments which accurately reflect the severity of the specific PMTS features.

It seemed that the least ambiguous results would be obtained by a study of women who were severely affected premenstrually yet had no clinical evidence of emotional or behavioral disturbance at other phases of their menstrual cycle. If PMTS could be defined in these women and the data used to construct operationally defined criteria and specific symptom rating scales, it should be possible to more reliably compare future studies of the etiology and treatment of this disabling condition.

\section{METHODS}

\section{Subjects}

Forty-two women suffering severe premenstrual symptoms were carefully selected for this study. Included were women between the ages of 22 and 43 years (mean 32) with no significant abnormal findings at physical examination and with normal laboratory values. They all had regular menses and were completely drug free for at least 4 weeks prior to and during the evaluation period. They reported premenstrual dysphoric symptoms for at least six consecutive menstrual cycles. Emotional as well as physical symptoms were present, monitored by various observer and self-rating scales, and rated at least 'moderate' to 'severe' during the premenstrual period with 'marked' or 'complete' relief with the onset of menses. With these strict inclusion criteria listed in Table 1, it was difficult to generate the population we required and only one out of six volunteers qualified. The group of 42 subjects eventually selected for the study were severely affected mature adult women most of whom were married with children. They were distinctly different from random groups of undergraduate 
Table 1. Criteria for inclusion

1. Premenstrual dysphoric symptoms for at least six preceding menstrual cycles.

2. Moderate to severe physical and psychological premenstrual symptoms.

3. Symptoms only during the premenstrual period with marked relief at onset of menses.

4. Age between 18 and 45 years.

5. Not pregnant.

6. No hormonal contraception.

7. Regular menses for six previous cycles.

8. No psychiatric disorder, normal physical examination and laboratory test profile.

9. No drugs for preceding four weeks.

10. Will not receive the following drugs during the study:

Anxiolytics

Diuretics

Hormones

Neuroleptics.

students or psychiatric outpatients, such as have been used in many previous studies of PMTS.

\section{Procedure and instruments}

There was an extended evaluation for each qualifying subject which permitted confirmation of the principal inclusion criteria: all women suffered a severe premenstrual dysphoria and the disturbance was an on/off phenomenon. At the first visit each woman participated in an open interview which was aimed at obtaining a spontaneous description of the disturbance, its natural history, the degree to which it was thought to interfere with her life, and any other aspects of psychosexual functioning which she thought worthy of comment. During subsequent visits, subjects completed self-rating scales and semistructured interviews, and met separately with the research nurse and a psychiatrist, who would then complete the observer-rating scales and record their clinical notes. Visits to our clinic were scheduled to coincide as closely as possible with two specific points in the menstrual cycle: 1) a follicular phase visit on Day 9 (Day 1 = onset of menses) and 2) a luteal phase visit on, or around, Day 26, but always 2-6 days premenstrual.

During these visits we used the following rating scales to measure the symptomatology and record the change occurring between the follicular and luteal phases: MDQ, MAACL, Visual Analogue Scale (VAS) (Aitken (1969), Maxwell (1978), State-Trait Anxiety Inventory (Spielberger et al. (1970)), and Hamilton (Hamilton (1960) and Carroll (Feinberg et al. (1979)) Depression Scales. The Menstrual Distress Questionnaire is a scale on which women can rate their experience of 47 symptoms, with a score of 1 to 6 , at various points in the menstrual cycle. The questionnaire has two forms, the Form-T (Today) on which each symptom is rated according to its presence on the day of completing the scale and the Form-A which requires a retrospective account of the experience of symptoms at specific points during the most recent menstrual 
cycle. The State-Trait Anxiety Inventory, Multiple Affect Adjective Check List, and Hamilton Depression Scale have been used and validated extensively. The Carroll Depression Scale is a self-rating instrument developed on this unit which utilizes yes/no responses to 52 questions to generate scores for items which corresponded to the 17-item Hamilton Depression Scale.

\section{Data analysis}

In addition to considering total scores on the various rating scales, we performed an item analysis of the Menstrual Distress Questionnaire, examining, for individual symptoms, the change in scores between follicular and premenstrual visits. Although the very broad range of items on the MDQ have not all been shown to be specific to severe PMTS, when focused on the premenstrual phase in an appropriately selected group of women the core features of PMTS are almost certainly contained in the data generated by this instrument.

Two subgroups were formed from the 42 women depending on their total score on the MDQ-T at the follicular visit. The division was placed at a score of 70 so that we could separately analyze the items for any women whose total scores on the MDQ in the follicular phase were noticeably higher than the normative values of 59,67 , and 58 , reported in three previous studies (Moos (1968), Gruba \& Rohrbaugh (1975), Rouse (1978)). Sixteen of our 42 subjects did record high follicular phase scores while 26 scored below the criterion level of 70 .

The analysis sought to rank order the items according to degree of change between visits for each individual subject, and the frequency of the response within the total population studied. Any of the $47 \mathrm{MDQ}$ items for which more than half of the subjects recorded a score change of less than two were excluded. A severity index score was derived for the remaining items by summing for each, the product of score change and the number of subjects recording that value, where the minimum score change considered was three. The theoretical maximum severity index score for any item would be $210(42 \times 5)$ if every woman scored 1 (no symptoms) at the follicular visit and 6 (most severe) at the premenstrual visit. The severity index scores were expressed as a percentage of the maximum possible in each group.

Because of the historical emphasis on depression as the most prominent aspect of PMTS (Smith (1975)) we analyzed the individual items in the Hamilton and Carroll Depression Scales to examine which items produced the increased totals seen at the premenstrual visit. Items were considered if more than half of the subjects changed their score on the Hamilton Depression Scale by more than one unit, or reversed their response on the Carroll Depression Scale at the premenstrual visit in the direction of increased dysphoria.

\section{RESULTS}

Total scores obtained with the Visual Analogue Scale and with the MDQ-T ratings at the follicular and premenstrual visits are presented in Fig. 1. Note that the mean for the MDQ-T went from 74 at the follicular phase to 135 

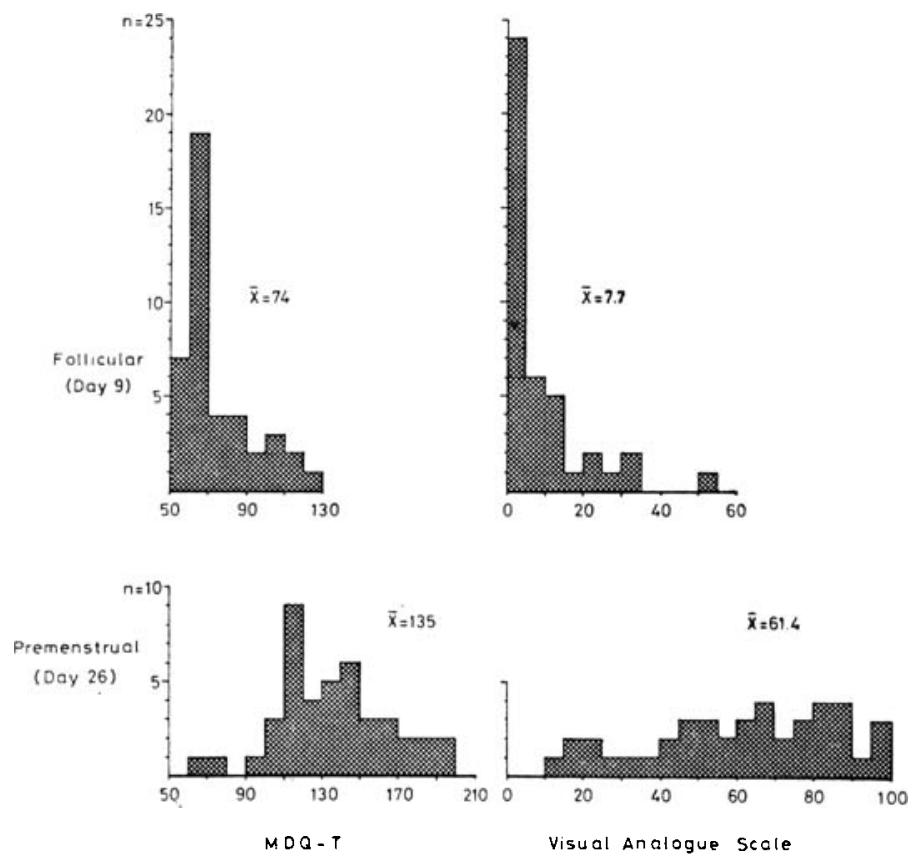

Fig. 1. Total scores obtained with the VAS and the $M D Q-T$ at the follicular and premenstrual visits $(\mathrm{n}=42)$.

premenstrually. These values can be compared with those obtained from the three previously studied populations (Moos (1968), Gruba \& Rohrbaugh (1975), Rouse (1978)), consisting of "wives of graduate students" "students of an introductory psychology course" and "visitors to a family planning clinic", who as already mentioned obtained follicular phase totals on the MDQ-T of 59, 67, and 58 respectively. The corresponding premenstrual values in those studies were 74,86 , and 85 . The markedly elevated mean premenstrual score of 135 for the women in the present study emphasizes the severity of their disturbance. The slightly higher mean value in the follicular phase, however, indicated that some of the subjects were recording significant symptoms at a visit when they claimed to be asymptomatic and fully recovered from their premenstrual disorder. The mean total score for the 26 women with low follicular MDQ-T scores (total $<70$ ) was 62 which is comparable with the normative data obtained from the three previous studies. The mean total for the follicular MDQ-T in the other 16 women was 93 which is obviously increased and suggests that despite our extensive clinical evaluation these women were suffering from a significant, albeit subclinical, degree of symptomatology at times other than the premenstruum. We therefore decided to include in the item analysis only the data from the 26 women with normative MDQ-T follicular scores. The premenstrual mean total MDQ score in these 26 women was 126. A rank-ordered 
Table 2. $M D Q$ individual item analysis of score change between follicular and premenstrual visits for 26 women with follicular $M D Q$ score $<70$

\begin{tabular}{|c|c|c|c|}
\hline $\begin{array}{l}\text { Rank } \\
\text { order }\end{array}$ & & Descriptor (MDQ item No.) & $\begin{array}{l}\text { Severity index } \\
\text { score (\%) }\end{array}$ \\
\hline 1 & & Irritability (36) & 52 \\
\hline 2 & & Mood swings (38) & 44 \\
\hline 3 & $\mathbf{P}$ & Swelling (e.g. abdomen, breasts, ankles) (34) & 42 \\
\hline 4 & & Restlessness (27) & 40 \\
\hline 5 & & Tension (45) & 40 \\
\hline 6 & & Depression (feeling sad or blue) (40) & 38 \\
\hline 7 & & Anxiety (21) & 38 \\
\hline 8 & $\mathbf{P}$ & Painful breasts $(30)$ & 36 \\
\hline 9 & & Difficulty in concentration (29) & 35 \\
\hline 10 & & Forgetfulness (6) & 35 \\
\hline 11 & & Crying (3) & 32 \\
\hline 12 & & Distractable (33) & 31 \\
\hline 13 & & Decreased efficiency (41) & 31 \\
\hline 14 & & Lowered judgement (24) & 30 \\
\hline 15 & & Change in eating habits (44) & 30 \\
\hline 16 & & Confusion $(7)$ & 29 \\
\hline 17 & $\mathbf{p}$ & Weight gain (1) & 28 \\
\hline 18 & & Fatigue (25) & 28 \\
\hline 19 & & Avoid social activities (20) & 27 \\
\hline 20 & & Lowered motor coordination (42) & 25 \\
\hline 21 & & Lowered school or work performance (4) & 23 \\
\hline 22 & $\mathbf{P}$ & Blind spots or fuzzy vision (46) & 22 \\
\hline 23 & & Feelings of well being (negative change) (31) & 21 \\
\hline 24 & & Take naps or stay in bed ( 8 ) & 20 \\
\hline 25 & & Accidents (e.g. cut finger, break dish) (35) & 20 \\
\hline 26 & & Affectionate (negative change) (13) & 15 \\
\hline 27 & & Loneliness (11) & 15 \\
\hline
\end{tabular}

$\mathbf{P}=$ physical symptom.

list of 27 MDQ items was obtained by the method described above and is presented in Table 2. Four of the items rank ordered as 3, 8, 17, 22 refer to physical ( $P$ ) changes and two describe positive affects ("feelings of well-being" and "affectionate" rank ordered at \# 23, 26 respectively). Disregarding the physical items, there are a total of 23 emotional and behavioral descriptors which show definite change between visits in a majority of the subgroup suffering solely from PMTS.

The group mean scores for the three subsets of the MAACL (anxiety, depression and hostility), the State Anxiety Inventory and Hamilton and Carroll Depression Scales, all changed significantly between follicular and premenstrual visits. No instrument appeared particularly sensitive to the changes of PMTS. Further details of these results are published elsewhere (Haskett et al. (1980)).

Item analysis of the Depression ratings was not productive for the Hamilton Scale. There was no item for which more than half of the subjects changed by more than one unit and there were only two items where more than one third of the subjects recorded such a change. These items were 7 ("work and 
Table 3. Item analysis of Carroll Depression Scale

\begin{tabular}{ccl}
$\begin{array}{c}\text { No. of } \\
\text { reversed responses }\end{array}$ & \multicolumn{1}{c}{ Descriptor (item No.) } \\
\hline 30 & - & I feel just as energetic as always (1) \\
28 & + & I feel irritable or jittery (31) \\
26 & - & My mind is as fast and alert as always (28) \\
24 & - & I feel in good spirits (32) \\
23 & + & I am restless and fidgety (10) \\
21 & - & I can concentrate easily when reading the papers (8) \\
\hline
\end{tabular}

$+=$ NO changed to YES

$-=$ YES changed to NO

interest") and 10 ("psychic anxiety"). The only dimension of endogenous depression which is presented in the premenstrual scores with the Hamilton Depression Scale is anhedonia, which by itself is not specific to depression.

The Carroll Depression Scale gave slightly higher values than the Hamilton Scale in the premenstruum but analysis showed that only six out of 52 items were prominent. These are listed in Table 3. The descriptors include "decreased energy", "slowed thinking" and "poor concentration". The second most common item is "irritability", and the remaining two items are "restlessness" and "not in good spirits". As for the Hamilton Depression Scale, the items recorded on the Carroll Depression Scale and contributing to the raised premenstrual score are not specific to endogenous depression and refer to similar emotional changes and decreased functioning as found on the MDQ.

\section{Construction of the diagnostic criteria and rating scales}

Based on a comprehensive review of the literature and our own clinical observations, we compiled a list of the major symptoms and signs of severe PMTS. These clinical features were combined with the rank-ordered lists of items from the MDQ and Carroll Scale. Using the patients' own words where possible, descriptors of the most characteristic aspects of this disorder were then incorporated into a list of operational criteria for PMTS. The format used is intentionally similar to that used by Spitzer et al. (1978) in the RDC and is shown in Fig. 2.

Subcategories ' $A$ ' and ' $B$ ' are devised using the item analysis and clinical interviews as shown in Table 4. ' $A$ ' contains descriptors which have been shown in this study to define the core changes which occur with PMTS. 'B' is a severity factor to ensure that only women who are severely affected will be included in this category. To obtain homogeneity in populations to be studied, it is necessary to distinguish minor variants of this disorder from the severe disabling condition. This is analogous to the need to separate individuals with minor mood fluctuations from those suffering from a major affective disorder in studies of that condition.

Many of the women in this study indicate that their symptoms of PMTS were not of identical severity in each menstrual cycle. Despite this apparent 
Table 4. $M D Q$ data base for proposed $R D C$

\begin{tabular}{rc}
\hline RDC item & Original MDQ item No. \\
\hline A -1 & 36 \\
2 & $21,27,45$ \\
3 & $8,25,41$ \\
4 & $3,31,38,40$ \\
5 & 35,42 \\
6 & $6,7,24,29,33$ \\
7 & 44 \\
$8^{*}$ & 4,20 \\
1 & \\
B -1 & \\
C $^{*}$ & \\
D $^{*}$ & \\
\hline
\end{tabular}

*Items included on the basis of clinical interviews and observations.

intercycle fluctuation, subcategory ' $C$ ' is included to ensure that subjects are suffering from an essentially recurrent condition.

Subcategory ' $D$ ' describes one of the major inclusion criteria which we used for this study. It emphasizes our suggestion that research into this condition must clearly define the particular time period under discussion. We do not believe that these symptoms are seen only in the premenstrual phase. There are many reports in the literature which suggest that variations of this complex are present at other phases of the menstrual cycle. We stress, however, the need to define this disturbance when it is confined to the premenstruum, before considering other syndromes which differ either in specific symptom configuration or temporal relationship.

The Primary/Secondary distinction is proposed for similar reasons to its inclusion in the criteria for Major Depressive Disorder. To facilitate research it is necessary to separate subjects who suffer from PMTS alone from those who manifest an interaction between other psychopathology and PMTS.

Using the same composite list of descriptors for this syndrome, self-report and observer-rating questionnaires were constructed. These are shown in Figs. 3 and 4 . It is intended that these scales should be complementary. The questionnaires are designed to monitor changes in the same specific PMTS phenomenology and the relationship between items is shown in Table 5 . 


\section{PRIMARY RECURRENT PREMENSTRUAL TENSION DISORDER}

This category is applied to female subjects in their fertile years who do not currently meet the criteria for any other psychiatric disorder.

The psychological and behavioral symptoms included in this disorder frequently occur in association with physical premenstrual symptoms, e.g. painful or tender breasts, headaches, swelling of abdomen, breasts or ankles, with water retention, weight gain, etc. These are not necessary for the psychiatric diagnosis.

A through $\mathrm{D}$ are required.

A. At least 5 of the following are required for definite and 4 for probable as part of a current episode.

1. Irritable, hostile, angry, short-fused.

2. Tense, restless, jittery, upset, high-strung, unable to relax.

3. Decreased efficiency, fatigue.

4. Dysphoric, marked spontaneous emotional lability, crying.

5. Lowered motor coordination, clumsy, prone to accidents (cut finger, break dish, etc.).

6. Distractable, confused, forgetful, difficulty in concentration, lowered judgement.

7. Change in eating habits (cravings, overeating, etc.).

8. Marked change in libido.

B. Overall disturbance is so severe that at least one of the following is present:

1. Serious impairment socially, with family, at home, at school or work.

2. Sought or was referred for help from someone or took medication (especially tranquillizers and/or diuretics) at least once during a premenstrual period.

C. Premenstrual dysphoric symptoms for at least the six preceding menstrual cycles.

D. Symptoms only during the premenstrual period with relief soon after onset of menses.

\section{SECONDARY RECURRENT PREMENSTRUAL TENSION DISORDER}

This category is applied for subjects who meet the criteria A through D for Primary Recurrent Premenstrual Tension Disorder but at the same time meet the criteria for another psychiatric disorder.

N.B. Some women previously given the diagnoses Intermittent Depressive Disorder, Minor Depressive Disorder and/or Labile Personality may now be more accurately classified as having Primary Recurrent Premenstrual Tension Disorder. 
Fig. 3. PMTS observer-rating scale

\begin{tabular}{ll}
\hline \multicolumn{2}{c}{ RATING SCALE FOR PREMENSTRUAL TENSION SYNDROME } \\
Name: & Rater: \\
Identification No.: & Date:
\end{tabular}

Circle the most appropriate score for each item:

1. Irritability - Hostility (0-4)

(Irritable, hostile, negative attitude, angry, short-fused, yelling and screaming at others)

0 . Not irritable.

1. Doubtful, trivial. Not reported without direct questioning.

2. Mild. Occasional outbursts of anger and hostile behavior. Spontaneously reported.

3. Moderate. Irritable behavior evident. Frequent outbursts.

4. Severe. Affects most interactions between patient and significant others.

\section{Tension (0-4)}

(Tense, restless, jittery, upset, highstrung, unable to relax)

0 . Not tense.

1. Doubtful, trivial.

2. Mild. Reports occasional tension.

3. Moderate. Tense, jittery, unable to relax. Restless behavior evident.

4. Severe. Constantly tense and upset.

\section{Efficiency (0-4)}

(Decreased efficiency, easily fatigued)

0 . No disturbance.

1. Doubtful, trivial.

2. Mild. Somewhat reduced efficiency.

3. Moderate. Easily fatigued, gets much less done than usual.

4. Severe. Fatigue causes serious interference with functioning.

4. Dysphoria (0-4)

(Dysphoric mood, distinguish from depression)

0 . Not dysphoric.

1. Somewhat blue, sad. Elicited only on direct questioning.

2. Mild dysphoric and labile mood, spontaneously reported.

3. Marked spontaneous emotional lability; occasional crying; feelings of loneliness.

4. Severe, obvious and persistent.
5. Motor coordination (0-4)

(Clumsy, prone to accidents, lowered motor coordination)

0 . No disturbance.

1. Doubtful, trivial.

2. Mild clumsiness, feels awkward.

3. Moderate. Frequent "accidents" while doing simple housework or on the job.

4. Severe impairment in motor coordination, e.g. unable to write properly, sew, or unable to drive.

6. Mental - cognitive functioning (0-4)

(Forgetful, poor concentration, distractable, confused, lowered judgement)

0 . No disturbance.

1. Doubtful, trivial.

2. Mild. Slight forgetfulness and distractability.

3. Moderate. Performance impaired by poor concentration, cognitive disorganization, forgetfulness, etc.

4. Severe. Marked deterioration in cognitive capacity, poor judgement, leading to regrettable decisions.

7. Eating habits (0-2)

0 . No change.

1. Mild increase in food intake, eating at odd, irregular hours, mostly snacks and sweets.

2. Obvious, marked increase. Uncontrollable cravings for sweets, chocolate, etc.

8. Sexual drive and activity (0-2)

0 . No change.

1. Mild but consistent increase or decrease in sexual drive, desire, libido.

2. Marked change in sexual drive with definite change in sexual behavior.

9. Physical symptoms (0-4)

(Painful or tender breasts; swelling of abdomen, breasts, ankles, or

(Fig. 3 continued on next page) 
fingers; water retention; weight gain; headaches, low-back pain, etc.)

0 . No physical symptoms.

1. Doubtful or trivial.

2. Mild. Some symptoms, increased awareness of bodily changes.

3. Moderate. Obvious changes and complaints.

4. Severe. Physical symptoms are incapacitating. Pain and discomfort. Marked water retention and edema. Weight gain more than $5 \mathrm{lbs}$.
10. Social impairment (0-4)

(Avoidance of social activities and interactions with family, at home, at work, at school, etc.)

0 . No social impairment.

1. Doubtful, trivial.

2. Mild avoidance of social activity.

3. Moderate but obvious impairment of social activity, mainly noticeable at home and with family.

4. Severe. Marked impairment of most social interactions including at work or school. Withdrawal, isolation.

Total score:

Fig. 4. PMTS self-rating scale

SELF-RATING SCALE FOR PREMENSTRUAL TENSION SYNDROME

Name:

Date:

Identification No.:

Instructions: The following questions are concerned with the way you feel or act today (or the way you felt or acted during the week).

Please answer all questions by circling YES or NO as indicated.

1. Do you find yourself avoiding some of your social commitments?

YES NO

2. Have you gained 5 or more pounds during the past week?

YES NO

3. Is your coordination so poor that you are unable to use kitchen utensils, garden tools or unable to drive?

4. Do you feel more angry than usual?

YES NO

5. Do you avoid family activities and prefer to be left alone?

YES NO

6. Do you doubt your judgement or feel that you are prone to hasty decisions?

YES NO

7. Do you feel more irritable than usual?

YES NO

8. Is your efficiency diminished?

YES NO

9. Do you feel tense and restless?

10. Do you feel a marked change in your sexual drive or desire during the last week?

If YES, is it increased or decreased?

YES NO

YES NO

YES NO

11. Are your present physical symptoms causing so much pain and discomfort that you are unable to function?

12. Have you recently cancelled previously scheduled social activities?

YES NO

13. Do you feel as if you were unable to relax at all?

14. Do you feel confused? $\quad$ YES NO

15. Do you suffer from painful or tender breasts?

YES NO

16. Do you have an increased desire for specific kinds of food (e.g. cravings for candy, chocolate, etc.)?

YES NO

17. Do you scream/yell at family members (friends, colleagues) more than usual? Are you "short-fused"?

YES NO

18. Do you feel sad, gloomy, and hopeless most of the time?

YES NO

(Fig. 4 continued on next page) 
19. Do you feel like crying?

YES NO

20. Do you have difficulty completing your daily household/job routine?

21. Was there a marked change in your sexual drive with definite change in your sexual behavior during the last week?

YES NO

22. Do you find yourself being more forgetful than usual or unable to concentrate?

YES NO

23. Do you happen to have more "accidents" with your daily housework/ job (cut fingers, break dishes, etc.)?

24. Have you noticed significant swelling of your breasts and/or ankles and/or bloating of your abdomen?

YES NO

YES NO

YES NO

YES NO

25 . Does your mood change suddenly without obvious reason?

26. Are you easily distracted?

27. Do you think that your restless behavior is noticeable by others?

28. Are you clumsier than usual?

29. Are you obviously negative and hostile towards other people?

30. Are you so fatigued that it interferes with your usual level of functioning?

YES NO

YES NO

YES NO

YES NO

YES NO

31. Do you tend to eat more than usual or at odd irregular hours (sweets, snacks, etc.)?

YES NO

YES NO

YES NO

YES NO

YES NO

YES NO

Table 5. Relationship of items on observer and self-rating scales

\begin{tabular}{ccccc}
\hline \multicolumn{2}{c}{ Observer-rating scale } & & \multicolumn{2}{c}{ Self-rating scale } \\
\cline { 2 - 2 } \cline { 5 - 6 } Item No. & Score & & Item No. & Score \\
\hline 1 & $0-4$ & & $4,7,17,29$ & $0-4$ \\
3 & $0-4$ & & $9,13,27,34$ & $0-4$ \\
4 & $0-4$ & & $8,20,30,32$ & $0-4$ \\
5 & $0-4$ & & $18,19,25,35$ & $0-4$ \\
6 & $0-4$ & & $3,23,28,33$ & $0-4$ \\
7 & $0-4$ & & $6,14,22,26$ & $0-4$ \\
8 & $0-2$ & & 16,31 & $0-2$ \\
9 & $0-2$ & & 10,21 & $0-2$ \\
10 & $0-4$ & & $2,11,15,24$ & $0-4$ \\
\hline Maximum total & $0-4$ & & $1,5,12,36$ & $0-4$ \\
\hline
\end{tabular}

\section{DISCUSSION AND SUMMARY}

Standard instruments for measuring severity and change in psychiatric disorders have failed to capture the essential symptom profile of PMTS. We used the MDQ as our data base and have reduced it from 47 to 27 core PMTS items by considering only those features which changed markedly in a majority of subjects from follicular to premenstrual visits. The study population was first refined to exclude those subjects whose symptom level in the follicular phase was higher than reported normative data. We defined the severe emotional and behavioral disturbance which occurs in some women, specifically during the premenstrual phase and in the absence of any ongoing physical or psychiatric 
pathology. Using rank-ordered items from existing rating scales, clinical observations and the subjects' descriptions of their feelings and symptoms, we have constructed diagnostic criteria and rating instruments for PMTS.

The strongest clinical items are presented in terms which we expect to be meaningful for women suffering from PMTS and therapists working with them. We have tried to avoid linguistic pitfalls which have confused the issue in the past. Specifically we learned that the MDQ items "anxiety" and "depression" were interpreted by many women as "tension" and "dysphoria" respectively. The same two items have been repeatedly used by clinicians to designate the syndrome as "Premenstrual Anxiety" and "Premenstrual Depression" when it is highly likely that women were using these emotional descriptors for quite different meanings. We believe that these terms are very misleading. PMTS does not appear to be an abbreviated form of an Anxiety Disorder nor is it a mini-episode of Endogenous Depression. The core symptomatology of PMTS includes irritability, dysphoria, restlessness, tension, and emotional lability. Additional features include fatigue, avoidance of social activity, decreased efficiency, difficulty concentrating, and occasional impairment of motor coordination. Symptoms of anxiety or depression, when present, seem to be reactive to the unexplained irritability and dysphoria.

As already mentioned in our previous report (Haskett et al. (1980)) no conclusion can be drawn from this study regarding the relationship between physical and emotional symptoms, since we used the presence of physical symptoms as part of our inclusion criteria. Our clinical observations support the view that some women manifest the emotional-behavioral PMTS without the physical manifestations. We decided to exclude the physical symptoms from the RDC, but they have been included in the rating scales so that this issue can be studied more closely in the future.

We feel that the use of these instruments will enable investigators of PMTS to more effectively compare data with each other. PMTS is disabling in its severe form and studies of its etiology and treatment have definite clinical significance. Although it does not appear to be a model for recurrent affective disorder, PMTS and its variations continue to provide intriguing subjects for the study of the biological substrates of specific behavior changes.

\section{ACKNOWLEDGEMENTS}

The authors are grateful to Mary Sies, Anna Babbitt, Mary Grix and Liz Romkema for their expert editorial assistance.

\section{REFERENCES}

Aitken, R. C. B. (1969): Measurement of feelings using Visual Analogue Scales. Proc. roy. Soc. Med. 62, 989-993.

Altman, M., E. Knowles \& H.D. Bull (1941): A psychometric study of the sex cycle in women. Psychosom. Med. 3, 199-225.

Coppen, A., \& N. Kessel (1963): Menstruation and personality. Brit. J. Psychiat. 109, 711-721.

Dalton, $K$. (1964): The premenstrual syndrome. C. C Thomas, Springfield, Illinois.

Diamond, S. B., A. A.Rubinstein, D. L. Dunner \& R. R. Fieve (1976): Menstrual problems in women with primary affective illness. Comprehens. Psychiat. 17, 541-548.

Feinberg, M., B. J. Carroll, P. Smouse, S.Rawson, R. F. Haskett, M. Steiner, A. Albala 
\& T. Zelnick (1979): Comparison of physician and self-ratings of depression. Abstract No. 80, Annual Meeting of Society of Biological Psychiatry, Chicago, Ill.

Fortin, J. N., E. D. Wittkower \& F. Katz (1958): A psychosomatic approach to the prementrual tension syndrome: A preliminary report. Canad. med. Ass. J. 79, 978981.

Frank, R. T. (1931): The hormonal causes of premenstrual tension. Arch. Neurol. Psychiat. 26, 1053-1057.

Green, R., \& K. Dalton (1953): The premenstrual syndrome. Brit. med. J. 1, 1007-1013.

Gruba,G.H., \& M. Rohrbaugh (1975): MMPI correlates of menstrual distress. Psychosom. Med. 37, 265-273.

Hamilton, M. (1960): A rating scale for depression. J. Neurol. Neurosurg. Psychiat. $23,56-62$.

Haskett, R. F., M. Steiner, J. N. Osmun \& B. J. Carroll (1980): Severe premenstrual tension - delineation of the syndrome. Biol. Psychiat. 15, 121-139.

Maxwell, C. (1978): Sensitivity and accuracy of the Visual Analogue Scale: A psychophysical classroom experiment. Brit. J. clin. Pharmacol. 6, 15-24.

Moos, R. H. (1968): The development of a Menstrual Distress Questionnaire. Psychosom. Med. 30, 853-867.

Moos, R. H. (1969): Menstrual Distress Questionnaire. Preliminary manual. Social Ecology Lab., Stanford, California.

Rees, L. (1953): Psychosomatic aspects of the premenstrual tension syndrome. Brit. J. Psychiat. 99, 62-73.

Rouse, P. (1978): Premenstrual tension: A study using the Moos Menstrual Questionnaire. J. Psychosom. Res. 22, 215-222.

Smith, S. L. (1975): Mood and the menstrual cycle. In Sachar,E.J. (ed.): Topics in psychoendocrinology. Grune \& Stratton, New York, pp. 19-58.

Smith, S. L., J.M. Cleghorn, D. L. Streiner \& E.V. Younglai (1975): A study of estrogens and progesterone in premenstrual depression. In: The family, 4th Int. Congr. of Psychosomatic Obstetrics and Gynecology, Tel Aviv, 1974. Karger, Basel, pp. 538-542.

Spielberger, C. D., R. L. Gorsuch \& R.E.Lushene (1970): STAI manual. Consulting Psychologists Press, Palo Alto, California.

Spitzer, R. L., J. Endicott \& E. Robins (1978): Research Diagnostic Criteria. Rationale and reliability. Arch. gen. Psychiat. 35, 773-782.

Steiner, M., \& B.J. Carroll (1977): The psychobiology of premenstrual dysphoria: Review of theories and treatments. Psychoneuroendocrinology 2, 321-335.

Zuckerman, M., \& B. Lubin (1965): Manual for the Multiple Affect Adjective Check List. Educational and Industrial Testing Service, San Diego, California.

American Psychiatric Association (1978): Diagnostic and statistical manual of mental disorders. 3rd ed. (draft), APA, Washington, D.C.

Commission on Professional and Hospital Activities (1973): H-ICDA, Hospital adaptation of ICDA, 2nd ed. Ann Arbor, Michigan.

Received April 8, 1980

Meir Steiner*, M.D., Ph.D.

Roger F. Haskett, M.B., B.S.

Bernard J. Carroll, M.B., Ph.D.

Department of Psychiatry

Mental Health Research Institute

University of Michigan

Ann Arbor, Michigan 48109

U.S.A.

* All correspondence to:

Shalvata Psychiatric Center

Tel Aviv University Medical School

POB 94, Hod-Hasharon

Israel 